\title{
Nodular Partial Response
}

National Cancer Institute

\section{Source}

National Cancer Institute. Nodular Partial Response. NCI Thesaurus. Code C103422.

Remission with remaining lymphoid nodules reflecting residual disease. 